\title{
Study of collective motion in liquid alkali metals
}

\author{
RAJESH C MALAN ${ }^{1, *}$ and ADITYA M VORA ${ }^{2}$ \\ ${ }^{1}$ Department of Applied Science and Humanities, Government Engineering College, (G.T.U.), Valsad 396001, India \\ ${ }^{2}$ Department of Physics, University School of Sciences, Gujarat University, Ahmedabad 380009, India \\ *Author for correspondence (rcmgecv@ gmail.com)
}

MS received 16 July 2018; accepted 17 December 2018; published online 4 May 2019

\begin{abstract}
Study of collective motion in the liquid alkali metals is provided through the current article. Phonon dispersion curves for liquid alkali metals are presented using a second order approach in conjunction with the Hubbard-Beeby (HB) equation. Both longitudinal and transverse components of the collective motion are obtained through the present model. A classical approximation approach of the pseudopotential is used to describe the core interaction of the electronic configuration. Screening as well as exchange and correlation effect are determined by local field correction functions given by Hartree (H), Ichimaru-Utsumi (IU) and Sarkar et al (S). Related parameters and constants are reported in line with the current computation to make the study comprehensive.
\end{abstract}

Keywords. Hubbard-Beeby approach; liquid alkali metals; phonon dispersion curves.

\section{Introduction}

\subsection{Preface}

Collective motion in liquid and in gas has been studied theoretically, through computer simulation and also experimentally since long time. Neutron scattering is the most common and effective experimental tool to determine phonon dispersion curves [1-13]. The study of the neutron scattering has provided evidence of some elementary excitation in the liquid metals. Starting from the milestone experiment carried out by Copley and Rowe [1] to Cabrillo et al [3] provides the insight into the dynamic properties of the alkali metals. Even before this experimental investigation, Desai and Nelkin [14] reported the collective motion in liquid argon theoretically.

\subsection{Broad methodology for phonon dispersion}

It is possible to find the phonon dispersion relation theoretically using force constant. But even for a regular and simple system like a simple cubic crystal, the required number of force constants is high. Therefore, for complex systems like liquid and amorphous solid phonon models are very important which describe the ion-electron interactions [15]. As the liquid metal is a highly correlating system, the random phase approximation cannot generate the actual picture of interaction. The collective motion in the liquid metals is very well explained by the Hubbard and Beeby (HB) [16]. Roughly, it can also be considered as a generalization of theory for the solid up to the liquid regime. In particular, to explain the phonon in liquid metals, a stepwise generalization of the phonon theory of the crystal has been made. In the first half of the procedure, it is to be extended to the disordered solid stage. In this stage, there is no relative atomic motion expected. In the latter half, it is to be extended to the continuous system with relative atomic motion i.e., liquid metals. Working in the same direction, the response function is to be approximated for the disordered system which is then converted to the nonstationary environment. To carry out work upon the formation of theoretical modes, the high degree of physics requires which includes kinetics along with the coupling of various modes [17-21]. These excitations are density-dependent and hence they follow, in some manner, the structure factor variations with respect to wave numbers. The structure factor peak position falls roughly at the minima of the longitudinal frequency $\left(\omega_{\mathrm{L}}\right) v s$. wave vector $(k)$ plot [22].

Unlike the conventional method, the current article uses a pair correlation $g(r)$ function rather than the structure factor directly.

\subsection{Literature survey}

Many researchers have reported a study of the collective motions in different types of materials so far [23-27]. The HB equation is used by Vora [28] with the core potential given by Gajjar et al [29] for the dynamic study of the alkali metals. The comparison with the experimental data in the said article shows the poor agreement which may be due to the selection of potential. The HB theory also used in conjunction with the charged hard sphere reference system by Thakor et al [30] for the metals: $\mathrm{Na}, \mathrm{Mg}, \mathrm{Al}$ and $\mathrm{Pb}$. The application of the same theory used for the phonon dispersion calculation of the $3 \mathrm{~d}$ transition metals by Thakor et al [31]. Pratap et al [32] have reported the phonon study of the glass and the liquid material. 
In their study, they include $\mathrm{Li}, \mathrm{Na}$ and a binary alloy of $\mathrm{Li}$ and $\mathrm{Na}$. To obtain results closer to experimental data, they add an extra term called the Born-Mayer term for the core [32]. In short, many studies related to the dynamics of the liquid metal and alloys have been used to study collective motions in the material.

Through the current article, an effort has been made to estimate the intensity and locate the positions of peaks of the longitudinal $\left(\omega_{\mathrm{L}}\right)$ and transverse $\left(\omega_{\mathrm{T}}\right)$ frequency with respect to the wave vector $k$. The pseudopotential theory has been applied to the current study. The well-behaved potential proposed by Fiolhais et al [33] used with its universal parameters $\alpha$ and $R$. It is also important to take into account the screening and the exchange and correlation effect, while calculating ion-electron interaction. The most suitable exchange and correlation functions are proposed for the potential to predict the excellent results. For the very first time, this much of local field correction functions are reported in the study of phonon dispersion relation with the said universal potential.

\section{Theory}

\subsection{Phonon dispersion relation}

As the basic ingredients of the calculation, two main quantities are to be obtained i.e., (a) the pair correlation function $g(r)$ and (b) the pair potential.

Instead of directly adopting the values of pair correlation function from the experimental data, it is computed as a prerequisite for further calculation. This makes the current prediction purely theoretical that does not depend on any experimental task performed earlier. The expression for the $g(r)$ used in this calculation is given below [34]:

$$
g(r)=\exp \left[\frac{-V(r)}{k_{\mathrm{B}} T}\right]-1
$$

where $k_{\mathrm{B}}$ is the Boltzmann constant and $T$ is the temperature. The whole calculation is made at temperature just above the melting point of each metal, respectively. The pair potential is assumed as the sum of the two components: one of them estimates the interaction among the ions which can be represented as $Z e^{2} / r$. The other is the potential-dependent term. The effective pair potential can be expressed using the following equation:

$$
V(r)=\left(\frac{Z^{2} e^{2}}{r}\right)+\frac{\Omega}{\pi^{2}} \int F(q) \frac{\sin (q r)}{(q r)} q^{2} d q .
$$

The function $F(q)$ can be represented in terms of bare ion potential $W(q)$ as given in the following equation:

$$
F(q)=\frac{-\Omega q^{2}}{16 \pi}|W(q)|^{2} \frac{\left[\varepsilon_{\mathrm{H}}(q)-1\right]}{\left\{1+\left[\varepsilon_{\mathrm{H}}-1\right][1-f(q)]\right\}} .
$$

$W(q)$ represents the potential without any screening or exchange and correlation effects. It estimates the effective interaction of electrons and ions. The terms $\varepsilon_{\mathrm{H}}$ and $f(q)$ are used to designate the Hartree dielectric response functions [28] and the exchange and correlation functions. Apart from the Hartree function $(\mathrm{H})$, in the current article, two different local field correction functions are used: one of them is given by Ichimaru-Utsumi (IU) [35] and another given by Sarkar et al [36].

It is difficult to find single model potential that can be used for the calculation of all properties of a material very well. The problem arises when a change in the physical phase occurs from solid to liquid or vice versa. In other words, the potential proposed for the solid state cannot generate the desired results for the liquid state for all properties. To overcome the situation, we used the potential given by Fiolhais et al [33]. The reason for the selection of this potential is its good transferability and efficiency for the study of liquid alkali metals and alloys as reported in our earlier articles [31-34].

Both longitudinal and transverse frequencies are generated in the liquid alkali metals. To find the peaks and the positions of the peaks, the following expressions are used from HB theory [16]:

$$
\begin{aligned}
& \omega_{\mathrm{L}}^{2}(q)=\omega_{\mathrm{E}}^{2}\left[1-\frac{\sin (q d)}{q d}-\frac{\cos (q d)}{q^{2} d^{2}}+\frac{6 \sin (q d)}{q^{2} d^{2}}\right], \\
& \omega_{\mathrm{T}}^{2}(q)=\omega_{\mathrm{E}}^{2}\left[1-\frac{3 \cos (q d)}{q^{2} d^{2}}-\frac{3 \sin (q d)}{q^{3} d^{3}}\right],
\end{aligned}
$$

where $\omega_{\mathrm{E}}^{2}$ is the maximum frequency and it depends on the pair potential $V(r)$ in the following manner:

$$
\omega_{\mathrm{E}}^{2}=\mu \int_{0}^{\infty} g(r) V^{\prime \prime}(r) r^{2} d r
$$

The constant $\mu$ can be given as follows:

$$
\mu=\frac{4 \pi \rho}{3 M} .
$$

\subsection{Elastic constants and parameters}

Apart from the phonon dispersion curves, the present work is also extended to find some constants and parameters which are necessary to provide a comprehensive bulk for the liquid alkali metals. The conventional formulae are used to find the said parameters.

\section{Results and discussion}

The potential parameters and other required constants are shown in table 1 . The potential parameters are directly 
Table 1. Input parameters and constants.

\begin{tabular}{lcccc}
\hline & \multicolumn{3}{l}{ Potential parameters [33] } & \\
Metal & $\alpha$ & $R$ & $\Omega_{0}(\mathrm{au})^{3}[28]$ & $Z(\mathrm{au})[28]$ \\
\hline $\mathrm{Li}$ & 3.549 & 0.361 & 144.9 & 1 \\
$\mathrm{Na}$ & 3.075 & 0.528 & 254.5 & 1 \\
$\mathrm{~K}$ & 2.807 & 0.745 & 481.4 & 1 \\
$\mathrm{Rb}$ & 2.748 & 0.824 & 587.9 & 1 \\
$\mathrm{Cs}$ & 2.692 & 0.920 & 745.5 & 1 \\
\hline
\end{tabular}

adopted from the article of Fiolhais et al [33]. Figure 1 shows the variation in longitudinal vibration with respect to the wave vector for all the liquid metals under study. The colour coding is applied to display the results for different local field correction functions. Up to the half of the first peak $q$-values, plots for all three local field correction functions are almost overlapping. This means that no screening effect is noted down up to this wave vector value. As compared to the results generated by Vora et al [23], the screening as well as exchange and correlation effects are less for the potential used than that used in [28]. This fact holds stability up to the all $q$ values shown in figure 1 . The current results suppress the $\omega_{\mathrm{L}}$ as compared to those shown in ref. [28]. Figure 1 also shows the current results with the experimentally available data $[1,23]$ whereever it is available. In the case of $\mathrm{Li}$ and $\mathrm{Rb}$, good agreement is obtained in compression with experimental data, whereas for $\mathrm{Na}$, our results are in reasonable agreement with those data. The source deviation of the present results from the experimental results lies in the parameters of the
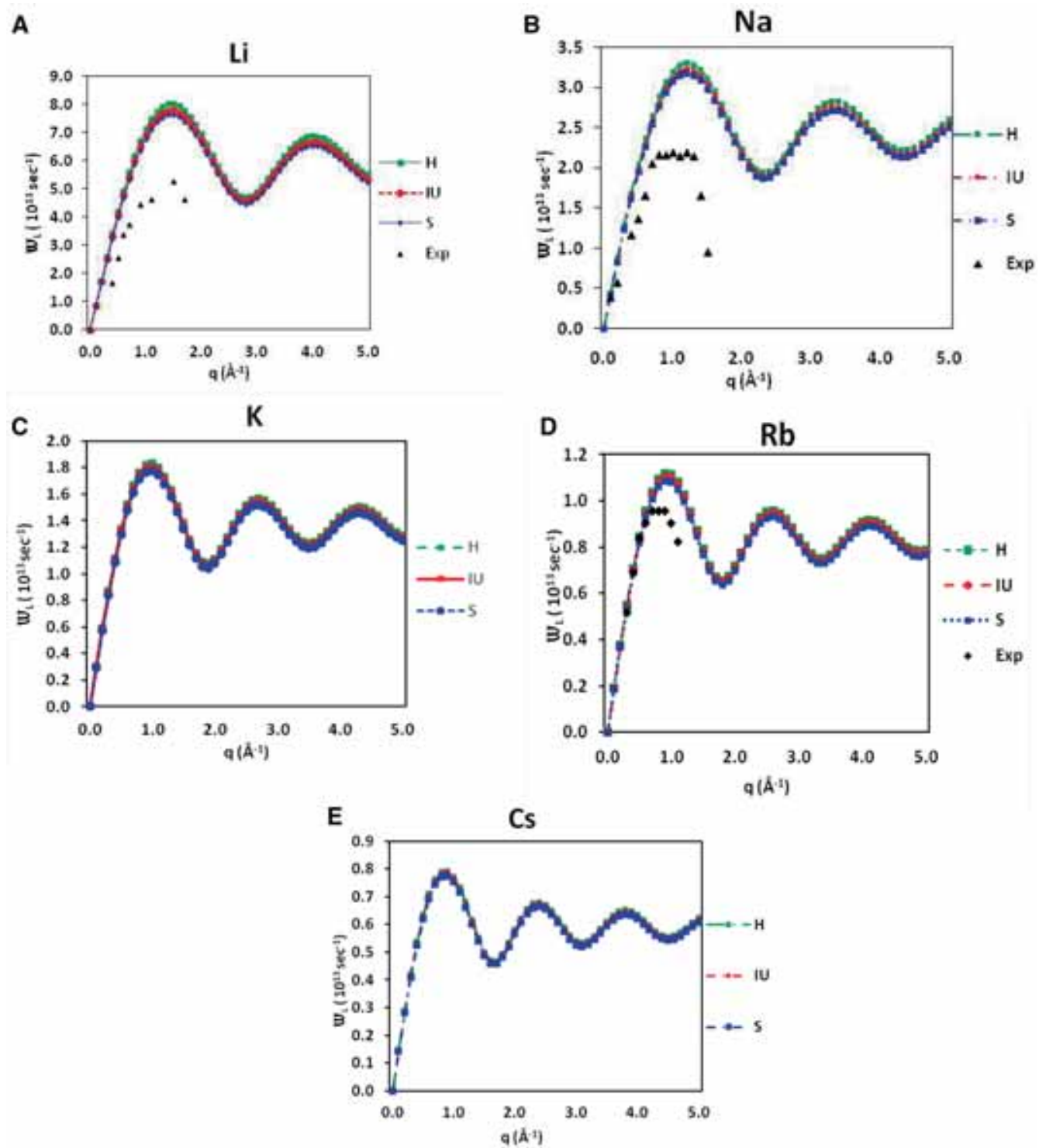

Figure 1. $\omega_{\mathrm{L}}$ for liquid (A) $\mathrm{Li},(\mathbf{B}) \mathrm{Na},(\mathbf{C}) \mathrm{K},(\mathbf{D}) \mathrm{Rb}$ and $(\mathbf{E}) \mathrm{Cs}$. 

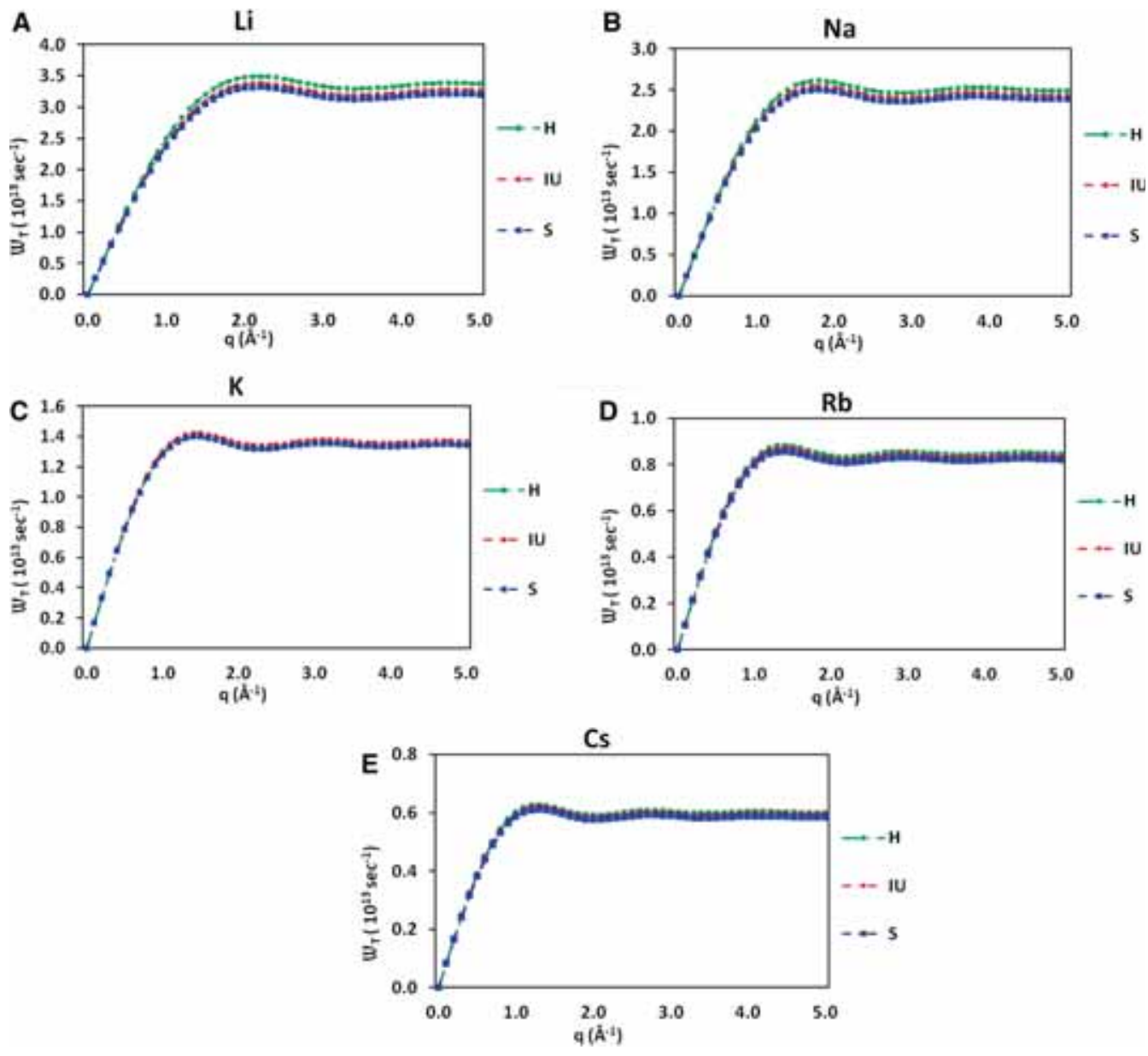

Figure 2. $\omega_{\mathrm{T}}$ for liquid (A) $\mathrm{Li},(\mathbf{B}) \mathrm{Na},(\mathbf{C}) \mathrm{K}$, (D) $\mathrm{Rb}$ and (E) Cs.

potential. As mentioned in the beginning of this section of the article, the present calculation uses the potential parameters directly as given by Fiolhais et al [33]. To maintain the originality of the potential and to meet the one of the goals of the present work of checking the transferability of the potential, no parameter fitting method is adopted. The original potential was proposed for the solid environment and the present work extends its application to the liquid metals. It affects the present results and creates some deviation from the experimental data. Still, it is apparently clear that the position of the peaks in all the three cases obtained very near to that of the experimental results.

In many of the theoretical researches, the results obtained for the calculation of the various properties of the alkali metal, the behaviour of $\mathrm{Li}$ shows poor agreement with respect to the expected results. While the current results remove this limitation for Li. This may occur because of the choice of the potential which describes the electron-ion interaction. The present combination of the HB approach [16] with the potential given by Fiolhais et al [33] adopted for the calculation of the longitudinal frequency generates good agreement irrespective of the metal type and its electronic configuration.
Figure 2 shows the transverse frequency response with respect to $q$ for all metals under the research. The influence of correction functions with respect to the static Hartree dielectric function $(\mathrm{H})$ is found very less for all the metals. While the Hartree function generates the higher value as compared to the other viz. S and IU for all the metals.

The aim to present the results in the form of figure 3 is to clear idea about the metal-wise comparison of the generated $\omega_{\mathrm{L}}$ and $\omega_{\mathrm{T}}$. Although it is possible to present the comparison for other two correction functions $\mathrm{S}$ and IU, as can be seen from figures 1 and 2 that the closeness of the results for all the three functions seems to be a repetition of the plots. Therefore, for the fruitful and short way to show the core idea is to present only one function (here, Hartree function $(\mathrm{H})$ ). As the atomic number changes, the highest value of the $\omega_{\mathrm{L}}$ and $\omega_{\mathrm{T}}$ decreases. This means that high-frequency oscillations are possible for $\mathrm{Li}$ and this value decreases while moving from $\mathrm{Li}$ to $\mathrm{Na}$. Thus, $\mathrm{Li}$ is highly transparent in the frequency propagation, whereas Cs is found to be the lowest in this case. In other words, it decreases while going from $\mathrm{Li} \rightarrow \mathrm{Na}$. Further, the shift in the position of the peak is observed from the higher $q$ value to the lower value, while going from $\mathrm{Li} \rightarrow \mathrm{Na}$. 

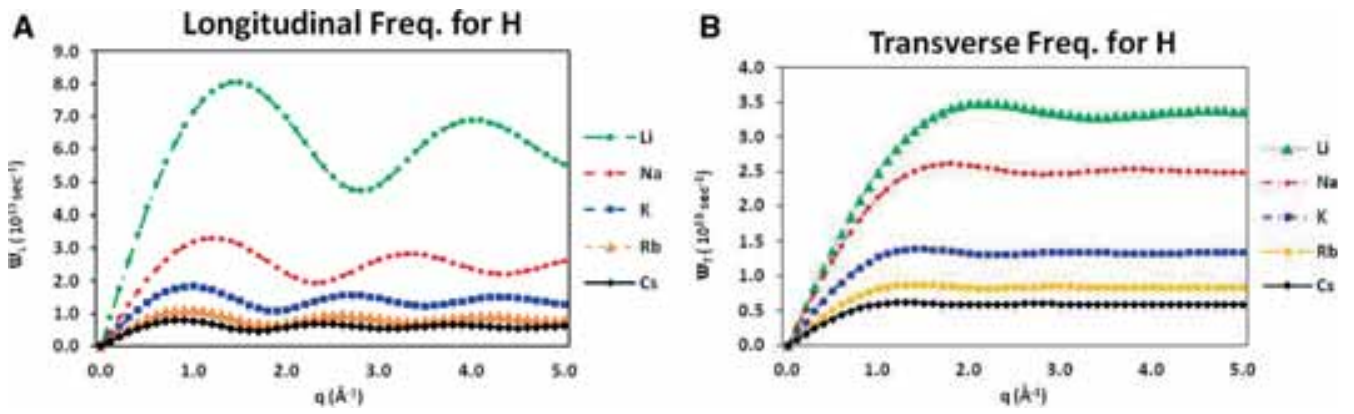

Figure 3. Metal-wise comparison for (a) $\omega_{\mathrm{L}}$ and (b) $\omega_{\mathrm{T}}$.

It is also to be observed that more oscillatory behaviour in the case of $\omega_{\mathrm{L}}$ is found as compared to $\omega_{\mathrm{T}}$ at higher $q$ values. This gives the surety of the collective oscillations due to only longitudinal phonons rather than both. The linear response is observed at the low $q$-value in phonon dispersion curves, whereas at higher $q$, this linearity disappears. This happens because the velocity is changed with the change in $q$. The HB method shows no discrepancy in oscillating behaviour for any metals under study and generates fairly good results.
The various types of parameters are also evaluated for all liquid metals as shown in table 2. The list of these constants includes the longitudinal velocity of sound $\left(V_{\mathrm{L}}\right)$, transverse velocity of sound $\left(V_{\mathrm{T}}\right)$, isothermal bulk modulus $\left(B_{\mathrm{T}}\right)$, modulus of rigidity $(G)$, Poisson's ratio $(\sigma)$, Young's modulus $(Y)$ and Debye temperature $\left(\theta_{\mathrm{T}}\right)$. The obtained results are shown in table 1 . The comparison with other's result and experimental data of the parameters is available only for Na. In case of Na, presently calculated parameters $\left(V_{\mathrm{L}}\right),\left(B_{\mathrm{T}}\right),(G)$ and

Table 2. Parameters computed in the study.

\begin{tabular}{|c|c|c|c|c|c|c|}
\hline Parameters & & $\mathrm{Li}$ & $\mathrm{Na}$ & K & $\mathrm{Rb}$ & Cs \\
\hline \multirow[t]{5}{*}{$V_{\mathrm{L}} * 10^{3} \mathrm{~cm} \mathrm{~s}^{-1}$} & $\mathrm{H}$ & 8.88 & 4.41 & 3.01 & 1.92 & 1.48 \\
\hline & IU & 8.61 & 4.31 & 2.97 & 1.90 & 1.47 \\
\hline & $\mathrm{S}$ & 8.44 & 4.22 & 2.92 & 1.87 & 1.44 \\
\hline & Others [37] & & 3.78 & & & \\
\hline & Expt. [38] & & 2.51 & & & \\
\hline \multirow[t]{5}{*}{$V_{\mathrm{T}} * 10^{3} \mathrm{~cm} \mathrm{~s}^{-1}$} & $\mathrm{H}$ & 5.13 & 2.54 & 1.74 & 1.11 & 0.85 \\
\hline & IU & 4.97 & 2.49 & 1.72 & 1.10 & 0.85 \\
\hline & $\mathrm{S}$ & 4.87 & 2.44 & 1.69 & 1.08 & 0.83 \\
\hline & Others [37] & - & 2.18 & - & - & - \\
\hline & Expt. [38] & - & 1.81 & 一 & - & - \\
\hline \multirow[t]{4}{*}{$B_{\mathrm{T}} * 10^{3}$ dyne $\mathrm{cm}^{-2}$} & $\mathrm{H}$ & 2.35 & 1.09 & 0.46 & 0.33 & 0.24 \\
\hline & IU & 2.21 & 1.04 & 0.45 & 0.33 & 0.24 \\
\hline & $\mathrm{S}$ & 2.12 & 1.00 & 0.43 & 0.32 & 0.23 \\
\hline & Others [37] & - & 7.35 & - & - & - \\
\hline \multirow[t]{4}{*}{$G$ dyne $\mathrm{cm}^{-2}$} & $\mathrm{H}$ & 14.10 & 6.54 & 2.76 & 2.01 & 1.45 \\
\hline & IU & 13.25 & 6.25 & 2.68 & 1.97 & 1.43 \\
\hline & $\mathrm{S}$ & 12.73 & 6.01 & 2.59 & 1.90 & 1.39 \\
\hline & Others [37] & - & 4.41 & - & - & - \\
\hline \multirow[t]{4}{*}{$\sigma$} & $\mathrm{H}$ & 0.25 & 0.25 & 0.25 & 0.25 & 0.25 \\
\hline & IU & 0.25 & 0.25 & 0.25 & 0.25 & 0.25 \\
\hline & $\mathrm{S}$ & 0.25 & 0.25 & 0.25 & 0.25 & 0.25 \\
\hline & Others [37] & - & 0.25 & - & - & - \\
\hline \multirow[t]{4}{*}{$Y * 10^{11}$ dyne $\mathrm{cm}^{-2}$} & $\mathrm{H}$ & 3.53 & 1.64 & 0.69 & 0.50 & 0.36 \\
\hline & IU & 3.31 & 1.56 & 0.67 & 0.49 & 0.36 \\
\hline & $\mathrm{S}$ & 3.18 & 1.50 & 0.65 & 0.47 & 0.35 \\
\hline & Others [37] & - & 1.10 & - & - & - \\
\hline \multirow[t]{3}{*}{$\theta_{\mathrm{T}}$} & $\mathrm{H}$ & 609.80 & 250.85 & 138.77 & 82.82 & 58.81 \\
\hline & IU & 591.30 & 245.12 & 136.93 & 82.0031 & 58.42 \\
\hline & $\mathrm{S}$ & 579.46 & 240.35 & 134.45 & 80.56 & 57.41 \\
\hline
\end{tabular}


$(Y)$ are in good agreement with the calculated results given in ref. [37], whereas it is in qualitative agreement with the experimental results [38] for $\left(V_{\mathrm{L}}\right)$ and $\left(V_{\mathrm{T}}\right)$. Variation from the other results may be due to the change in the potential that used in the current study, whereas the variation from the experimental data may be due to several reasons including the experimental environment.

\section{Conclusion}

Finally, it can be concluded that the HB method is found to be suitable for the study of the phonon dispersion curves for liquid alkali metals. In a collective manner with our previous articles [39-42] for the study of the liquid alkali metals the current results prove the potential as unique that can be used for the comprehensive study of the said group of liquid metals breaking the limitation of pseudopotential theory. The agreement between the obtained results and the experimental ones shows the efficiency of the current approach of the study.

\section{Acknowledgements}

AMV has acknowledged the computer facility developed under the DST-FIST programme from DST, Government of India, New Delhi, India and financial assistance under DRSSAP-I from UGC, New Delhi, India.

\section{References}

[1] Copley J R D and Rowe J M 1974 Phys. Rev. Lett. 3249

[2] Morkel C and Glaser W 1986 Phys. Rev. A 333383

[3] Cabrillo C, Bermejo F J, Alvarez M, Verkerk P, Maira-Vidal A, Bennington S M et al 2002 Phys. Rev. Lett. 89075508

[4] Novikov A G, Savostin V, Shimkevich A L, Yulmetyev R M and Yulmetyev T R 1996 Physica B 228312

[5] Verkerk P, De Jong P H K, Arai M, Bennington S M, Howells W S and Taylor A D 1992 Physica B 834180

[6] Balucani U, Torcini A, Stangl A and Morkel C 1996 J. NonCryst. Solids 205299

[7] Chieux P, Dupuy-Philon J, Jal J F and Suck J B 1996 J. NonCryst. Solids 205370

[8] Morkel C, Gronemeyer C, Glaser W and Bosse J 1987 Phys. Rev. Lett. 581873

[9] Pratesi G, Suck J B and Egelstaff P A 1999 J. Non-Cryst. Solids 91250

[10] Stangl A, Morkel C, Balucani U and Torcini A 1996 J. NonCryst. Solids $\mathbf{2 0 5} 402$

[11] De Jong P H K, Verkerk P and De Graaf L A 1994 J. Phys. Condens. Matter 68391
[12] Novikov A G, Savostin V, Shimkevich A L and Zaezjev M V 1997 Physica B 359234

[13] Morkel C and Bodensteiner T 1990 J. Phys. Condens. Matter 2251

[14] Desai R C and Nelkin M 1966 Phys. Rev. Lett. 16839

[15] Bilz H and Kress W 1979 Phonon dispersion relations in insulators (Berlin, Heidelberg: Springer)

[16] Hubbard J and Beeby J L 1969 J. Phys. C: Solid State Phys. 2 556

[17] Sjogren L and Sjolander A 1979 J. Phys. C 124369

[18] Bove L E, Dorner B, Petrillo C, Sacchetti F and Suck J B 2003 Phy. Rev. B 68024208

[19] Sjogren L 1980 J. Phys. C 13705

[20] Wahnstrom G and Sjogren L 1982 J. Phys. C 15401

[21] Berne B J 1977 Statistical mechanics (New York: Plenum Press)

[22] Bhatia A B and Singh R N 1985 Phys. Rev. B 314751

[23] Choudhari N, Rao K R and Chaplot S L 1991 Bull. Mater. Sci. 14959

[24] Achar B N N and Liu C 1991 Bull. Mater. Sci. 14973

[25] Agrawal B K, Agrawal S, Yadav P S, Kumar S, Nagi J S and Varshney N 1991 Bull. Mater. Sci. 14967

[26] Sadaiyandi K and Ramachandran K 1994 Bull. Mater. Sci. 17 141

[27] Thakore B Y, Gajjar P N and Jani A R 2000 Bull. Mater. Sci. 235

[28] Vora A M 2010 J. Non Oxide Glasses 291

[29] Gajjar P N, Patel M H, Thakore B Y and Jani A R 2002 Commun. Phys. 1281

[30] Thakor P B, Gajjar P N and Jani A R 2009 Pramana-J. Phys. 721045

[31] Thakor P B, Sonavane Y A, Gajjar P N and Jani A R 2011 AIP Conf. Proc. 1393163

[32] Pratap A, Lad K N and Raval K G 2004 Pramana-J. Phys. 63 431

[33] Fiolhais C, Perdew J P, Armster S Q, MacLaren J M and Brazczewska M 1995 Phys. Rev. 5114001

[34] Faber T E 1972 Introduction to the theory of liquid metals (London: Cambridge University Press)

[35] Ichimaru S and Utsumi K 1981 Phys. Rev. B 247358

[36] Sarkar A, Sen D, Haldar S and Roy D 1998 Mod. Phys. Lett. B 12639

[37] Patel V N, Thakor P B, Thakore B Y, Gajjar P N and Jani A R 2006 Condens. Matter Phys. 9741

[38] Bryk T and Mryglod I 2000 J. Phys. Condens. Matter 12 6063

[39] Malan R C and Vora A M 2018 in Taylor J C (ed.) Advances in chemistry research (New York: Nova Science Publishers vol. 42) p 245

[40] Malan R C and Vora A M 2018 AIP Conf. Proc. 1953 140014

[41] Malan R C and Vora A M 2018 J. Nano Elect. Phys. 10 0320-1

[42] Malan R C and Vora A M 2018 Int. J. Sci. Res. Rev. 7385 